\title{
Viscoelastic Combining Flow in a T-shaped Tube
}

\author{
By Kiyoji Nakamura*, Taro Nishimura**, Ichiro Miyahara*, \\ Takashi Noda* and Akira Horikawa*, Members TMSJ \\ * Faculty of Engineering, Osaka University, Suita, Osaka \\ ** Faculty of Textile Science, Kyoto Institute of Technology, \\ Sakyoku, Kyoto
}

Based on the Journal of the Textile Machinery Society of Japan, Transactions, Vol.39, No.9, T141-148 (1986)

\begin{abstract}
A viscoelastic combining flow in a T-shaped tube is examined using polymer (polyacrylamide) solutions. Streamlines near the combining part were observed from the two- or three-dimensional standpoint of view. Upstream of the combining part, circulating secondary flows were observed in the T-shaped tube as well as in a T-shaped channel composed of parallel plates. The streamline length changes according to the growth of the circulating secondary flow. Curves of streamline length plotted with Weissenberg number present an elastic effect of viscoelastic liquid and nearly become one straight line for the various throttling ratios and polymer concentrations. Hence, the elastic property of polymer solution contributes to the growth of the circulating secondary flow for the T-shaped combining tube flow.
\end{abstract}

\section{Introduction}

A flow in channel-connected two rectangular conduits has been studied, and the properties of viscoelastic dividing and combining flow in the channel were reported ${ }^{[1]}$. In this paper, a viscoelastic tube flow in a T-shaped pipe is discussed. Such a flow is observed frequently in a spinneret for conjugate spinning.

Many reports have been published on tube flows in regard to dividing blood flows ${ }^{[2]-[5]}$, and on the relation between loss energy and dividing-recombining flows by Ito et al. ${ }^{[6],[7]}$. But most of them are at high Reynolds numbers, and on Newtonian flows.

On viscoelastic flows, there are many reports with respect to circulating secondary flows ${ }^{[8]-[14]}$ having a contraction, the flow direction in which is the same at upstream and downstream.

Velocity distributions of viscoelastic tube flows were reported with polymer aqueous solutions ${ }^{[15]-[18]}$, but no reports are found concerning the upstream of a combining tube.

In the present paper, observation of viscoelastic combining flow in a T-shaped tube is described. Discussion is also with the flow differences between viscoelastic and Newtonian, and with the influences of viscoelasticity on tube flow.

\section{Experiments}

\subsection{Apparatus}

Two methods were used to visualize the tube flow. One is a two-dimensional observation method by adding

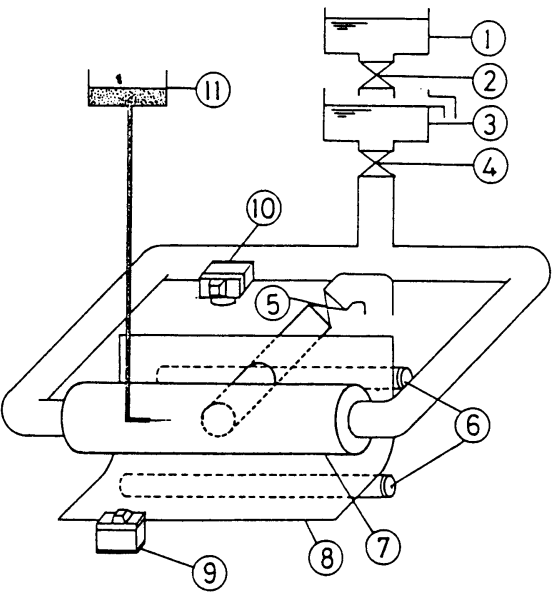

Fig. 1 Schematic diagram for flow measurements. (1) reservoir tank, (2), (4), (5) valves, (3) over flow tank, (6) light source, (7) test section, (8) scattering screen, (9), (10) camera, (11) tracer tank

aluminum powder, and the other is a three-dimensional method by mingling a stream of colored fluid.

Shown in Fig. 1 is a schematic diagrams of an experimental apparatus. Constant pressure head is kept by over flow from lower reservoir (3). Bulbs (2), (4), (5) regulate the flow rate. Light from sources (6) illuminates experimental tube (7) uniformly through scattering screen (8). A colored streamline issued from tracer tank (11) is photographed with two cameras (9), (10) set along the $z$ and the $y$ axes.

Shown in Fig. 2 is the dimension and coordinates of 


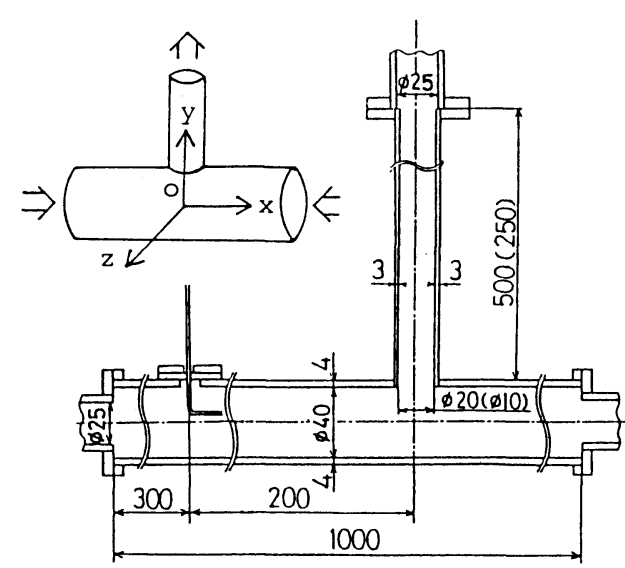

Fig. 2 Dimension and coordinates of T-shaped conduit

the T-shaped tube. The origin of the coordinates is a intersection of two axis of the tubes. Fluid enters from both ends of a $40 \mathrm{~mm}$ diameter inlet tube, and leaves to a $20 \mathrm{~mm}$ diameter outlet tube. Each length of the tube-branches is $500 \mathrm{~mm}$, long enough to ignore the inlet effect.

For two dimensional measurement, a light source is set at the place of camera (9), and a slit light is cast along $y$ axis to the center of the tube. Photographs are taken in $z$ direction with camera (10). For three dimensional measurement, the flow-in position of colored fluid is set at six points on $y-z$ plain shown in Fig. 3 about 200 $\mathrm{mm}$ apart from the combining part.

Observed area of the tube flow is within $100 \mathrm{~mm}$ from the combining part. In place of the $20 \mathrm{~mm}$ tube, an outlet tube of $10 \mathrm{~mm}$ wide and $250 \mathrm{~mm}$ long was used for another test. The velocity ratio between flow-in and flow-out, that is throttling ratio, is 8 for $20 \mathrm{~mm}$ tube and 32 for $10 \mathrm{~mm}$ tube.

Four working fluids were selected, namely a Newtonian glycerin of $1.7 \mathrm{~Pa} \cdot \mathrm{s}$ in viscosity and three viscoelastic polyacrylamide (SEPARAN-AP 30 : PAA) aqueous solutions of $0.1 \%, 0.4 \%$ and $0.8 \%$ in polymer concentration. Colored fluid was prepared by mixing the working fluid and dyes to avoid the effect of

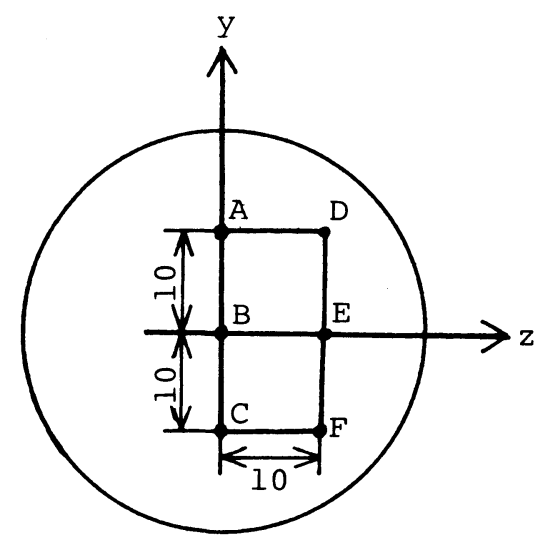

Fig. 3 Flow-in position of tracer fluid buoyancy.

\subsection{Correction to position of observed streamline}

Position of the observed streamline is distorted due to the difference of the refractive index between the liquid and the plastic material forming the tube, and the air. In the case of $x-y$ plain observation by slit lighting, the true location can be calculated from the observation along $z$ axis and refractive indices because the tracer locates on $x-y$ plain. But when the streamline locates apart from the tube axis, the true position must be decided by observation of the streamline from two different directions like (1) and (2) shown in Fig. 4. The tracer located at $a$ in the tube of radius $R$ is observed at $a^{\prime}$. Position $a$ is an intersection of lines $a c$ and $a b$. Line $a c$ or $a b$ can be obtained from the relation shown

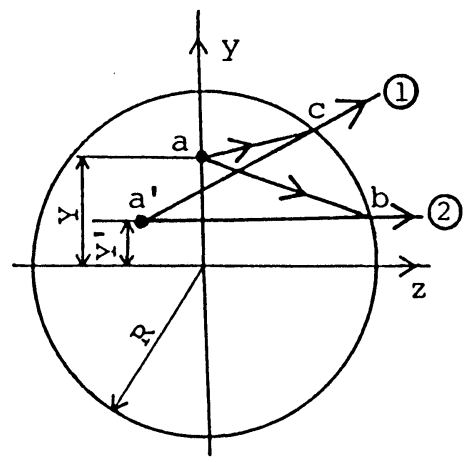

Fig. 4 Light path from a tracer and observed position

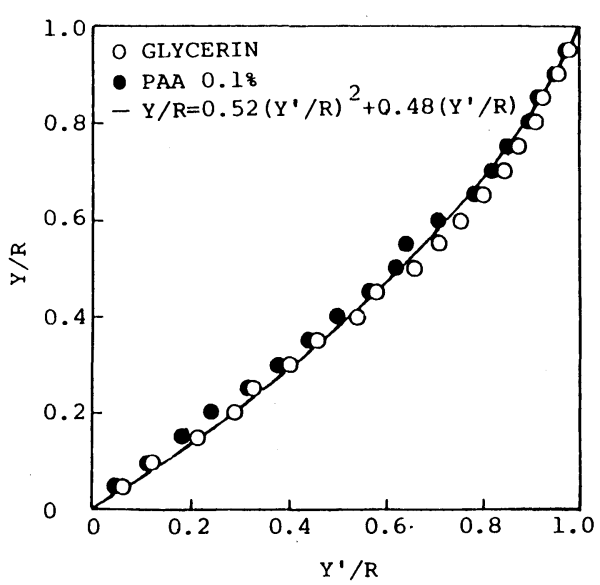

Fig. 5 Relation between observed position and true position of tracer

in Fig. 5 for PAA $0.1 \%$ aqueous and glycerin. Since the relation has little differences between liquids, the correction of tracer position is carried out with the solid line expressed by

$$
Y / R=0.52\left(Y^{\prime} / R\right)^{2}+0.48 Y^{\prime} / R
$$




\section{Swelling of Streamlines at Combining Part}

\subsection{Streamlines in vertical section at tube center}

Shown in Fig. 6 is observation of viscoelastic flow by means of slit light in vertical section at the tube center $(x-y$ plain shown in Fig. 1). When the mean velocity at the inlet tube is denoted by $v$, Reynolds number is

$$
R_{e}=\frac{D v \rho}{\eta} \ldots \ldots \ldots \ldots \ldots \ldots
$$

where $D$ is the inlet tube diameter, $\rho$ the density of liquid, and $\eta$ is the viscosity obtained from observation by Bewersdorff ${ }^{[19]}$ in which the shear rate is adapted at the position where the flow rate assuming viscous flow equals to mean velocity.

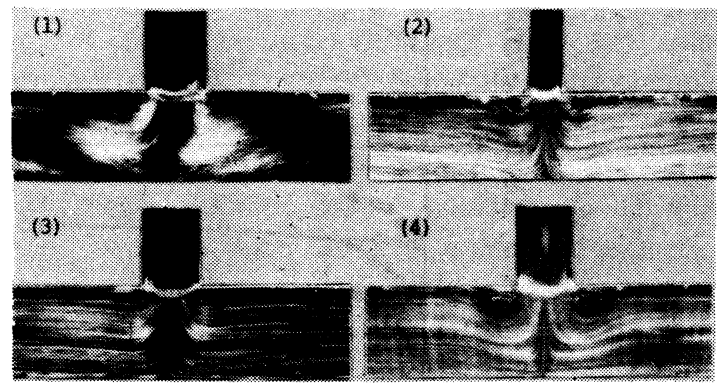

Fig. 6 Streamlines for PAA solution in the vertical section through the tube center

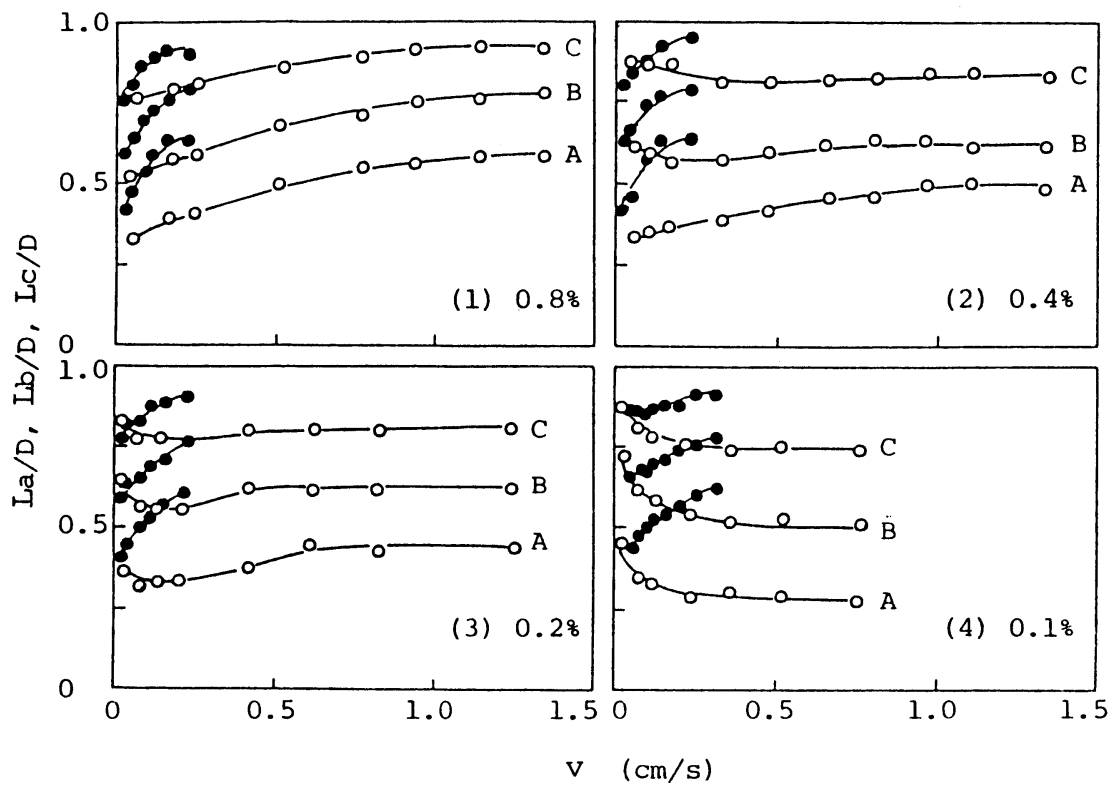

Fig. 8 Relation between flow rate and peak distance of swelling streamlines with throttling ratio $8(0)$ and $32(\bullet)$
In the case of slow flow shown (1) in Fig. 6, streamlines swell gradually already far from the combining part. But at high throttling ratio or high polymer concentration shown in (2) or (3), streamlines swell suddenly at the combining part. Swelling becomes large with increasing flow rate due to the growth of circulating secondary flow at the combining corner as shown (4). Far from the combining part, the streamlines are parallel to the tube axis ( $x$ axis). This shows that streamlines are swelled sharply by the existence of circulating secondary flow at the combining part when the fluid is subjected to high throttling ratio or has high polymer concentration. This is the result by non-Newtonian viscosity or elasticity of the fluids.

In order to evaluate this swelling, three peak distances between the wall and the maximum swellings of three tracer lines $A, B, C$ shown in Fig. 7 were measured as $L_{a}, L_{b}, L_{c}$. The positions of $A, B, C$ divide the tube diameter equal to a quarter. Shown in Fig. 8 is the relation between the peak distance and the flow rate with various polymer concentration and with two throttling ratios. When the throttling ratio is 8 , the values of three peak distances are increased with increasing flow rates for $0.8 \%$ concentration, suggesting the growth of swelling. Two peak curves of $B$ and $C$ for $0.4 \%$ concentration and three peak curves for $0.2 \%$

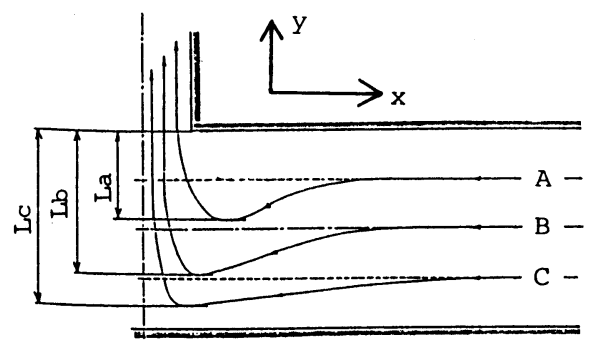

Fig. 7 Schematic diagram for swelling of streamlines 
have the minimum, suggesting the swelling growth at slow or fast flow rate. For low $(0.1 \%)$ concentration the swelling is observed only at low flow rate and the curves go down slowly with increasing flow rate.

When the throttling ratio is 32 , all peak curves with each concentration are increased with increasing flow rate. These curves are steeper than those for throttling ratio of 8 .

As describe above, in the case of low elasticity and low polymer concentration, the swelling was observed with slow flow or small throttling ratio. When the flow has no swell, the energy consumption is small for the stream in the vertical section of the tube center as well as for the stream apart from this section. But any stream must swell near the joint. So the total energy consumption will become smaller when the stream in the center vertical section swells. With increasing flow rate, the swelling disappears because of inertial effect. In the case of high polymer concentration (strong shear thinning property and high elasticity) or large throttling ratio (large deformation), viscoelastic property affects the combining field, and the circulating secondary flow grows to make the swelling enlarge at the combining corner.

3.2 Three dimensional movement of streamlines

At the combining part of the $\mathrm{T}$-shaped tube, the streamlines move three dimensionally except in the vertical section through the tube center. Shown in Fig. 9 are the observations of the stream traces starting from

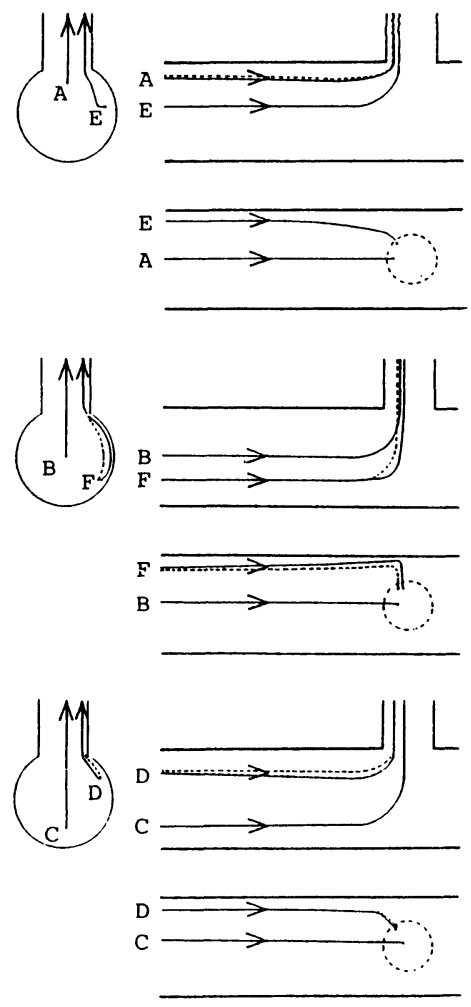

Fig. 9 Streak line of Newtonian fluids using glycerin
Table 1 Flow rate $(\mathrm{cm} / \mathrm{s})$ and Reynolds number for Fig. 9

\begin{tabular}{ll|lll}
\hline & & solid & \multicolumn{2}{c}{ dotted } \\
\hline \multirow{2}{*}{$A$} & $v$ & 0.21 & 0.44 & 0.58 \\
& $R e$ & 0.06 & 0.13 & 0.18 \\
\hline \multirow{2}{*}{$B$} & $v$ & 0.16 & 0.38 & 0.50 \\
& $R e$ & 0.05 & 0.11 & 0.15 \\
\hline \multirow{2}{*}{$C$} & $v$ & 0.16 & 0.23 & 0.42 \\
& $R e$ & 0.05 & 0.07 & 0.13 \\
\hline \multirow{2}{*}{$D$} & $v$ & 0.10 & 0.22 & 0.48 \\
& $R e$ & 0.03 & 0.07 & 0.14 \\
\hline \multirow{2}{*}{$E$} & $v$ & 0.11 & 0.27 & 0.48 \\
& $R e$ & 0.03 & 0.08 & 0.14 \\
\hline \multirow{2}{*}{$F$} & $v$ & 0.11 & 0.17 & 0.50 \\
& $R e$ & 0.03 & 0.05 & 0.15 \\
\hline
\end{tabular}

the six points for Newtonian liquid of glycerin. Mean velocity ( $v$ ) and Reynolds number $(R e)$ for each measurement are shown in Table 1. Two stream traces of larger flow rate are piled up one another as shown by a dotted line. Two stream traces started from $A$ and $B$ swell when approaching to the combining part. This is the same phenomena as the case of low polymer concentration and slow flow rate (small Reynolds number). Other two stream traces $D$ and $E$ near the wall move to $z$ direction already further from the combining part than the viscoelastic flow described in the next paragraph, and make swell the streamlines such as traces $A$ or $B$ in the vertical section through the tube center.

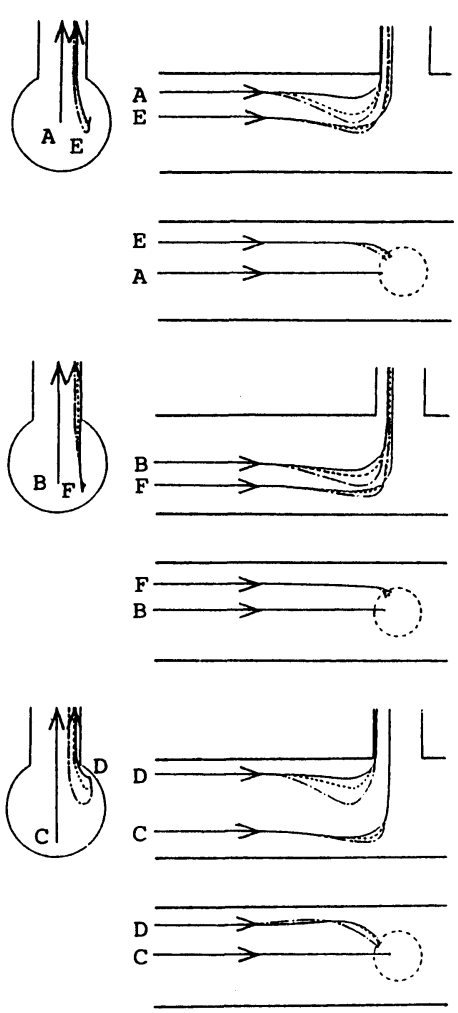

Fig. 10 Streak line of viscoelastic fluids using PAA $0.4 \%$ solution 
Table 2 Flow rate $(\mathrm{cm} / \mathrm{s})$ and Reynolds number for Fig. 10

\begin{tabular}{ll|ccc}
\hline & solid & dotted & broken \\
\hline \multirow{2}{*}{$A$} & $v$ & 0.12 & 0.42 & 0.85 \\
& $R e$ & 0.03 & 0.17 & 0.51 \\
\hline \multirow{2}{*}{$B$} & $v$ & 0.14 & 0.41 & 0.80 \\
& $R e$ & 0.03 & 0.17 & 0.47 \\
\hline \multirow{2}{*}{$C$} & $v$ & 0.13 & 0.37 & 0.72 \\
${ }^{*} R e$ & 0.03 & 0.14 & 0.40 \\
\hline \multirow{2}{*}{$D$} & $v$ & 0.13 & 0.36 & 0.85 \\
& $R e$ & 0.03 & 0.14 & 0.51 \\
\hline \multirow{2}{*}{$E$} & $v$ & 0.13 & 0.37 & 0.72 \\
& $R e$ & 0.03 & 0.14 & 0.40 \\
\hline \multirow{2}{*}{$F$} & & 0.16 & 0.47 & 0.85 \\
& $R e$ & 0.04 & 0.21 & 0.51 \\
\hline
\end{tabular}

With increasing flow rate (Reynolds number) the position at which the stream near the wall changes the direction approaches to the exit, and the swell on the tube center becomes small.

Stream traces for viscoelastic flow of PAA $0.4 \%$ solution are shown in Fig. 10, and their flow conditions are in Table 2. The swell of stream traces started from $A, B$ and $C$ becomes larger with increasing velocity as predicted by the observation of the secondary flow in the vertical section through the tube center. Size of the swell and its change rate are larger near the exit tube, that is, become smaller in order of $A, B$ and $C$.

For the stream traces started near the wall, namely from $E, F$ and $G$, the swells become larger with increasing flow rate as similar to the traces from $A, B$ and $C$ on the tube center. Size and the change rate of the swell of the trace $D$ near the exit are larger than those of the other $E$ or $F$. This tendency is also similar to that on the tube center.

The movement to $z$ direction seen with traces has begun already with increasing flow rate. This is because that the circulating secondary flow grows at the entrance of the exit tube, and obstructs the streams at the combining part. This suggests that the secondary flow observed on $x-y$ plane exists widely on the circumference of the entrance of the outlet tube.

\subsection{Length of streamline}

The shape of streak lines drawn by six stream traces depends on the rheological properties of liquid and the flow rate. The variation of the shape is shown by the length of the streak line in the fixed space as shown in Fig. 11. Line $L_{1}$ is a tangent of the stream trace parallel to the inlet tube axis, and its length is measured from an arbitrary point to the cross point with line $L_{2}$. Line $L_{3}$ is that parallel to the outlet tube, and the length is measured from its cross point with line $L_{2}$ to an arbitrary point. Line $L_{2}$ is parallel to $z$ axis and intersects with lines $L_{1}$ and $L_{3}$. Its length is from the cross point with $L_{1}$ to that with $L_{3}$. The above two arbitrary points are determined by setting the value of $L_{1}$ about $100 \mathrm{~mm}$

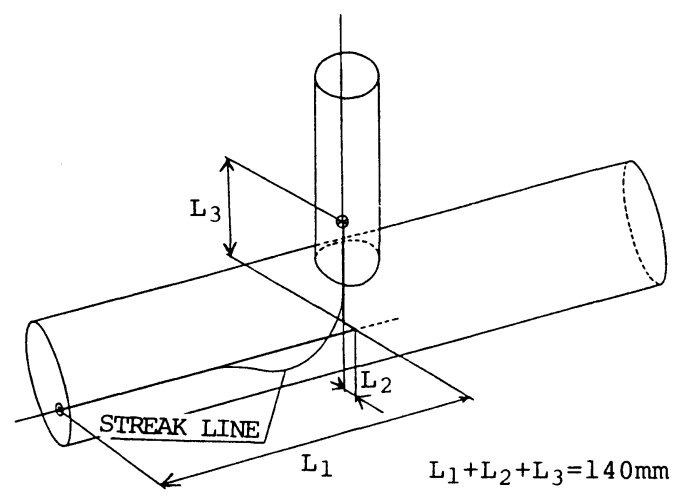

Fig. 11 Schematic diagram for streak line measurements

and the sum $L$ of $L_{1}, L_{2}$ and $L_{3}$ is $140 \mathrm{~mm}$ long. The length of the streak line, $l$, is measured in the space of this $140 \mathrm{~mm}$.

Shown in Fig. 12 is the length of the streak line started from $E$. The ordinate indicates the ratio of streak line to the fixed length $l / L$. In Fig. 12(1) the length ratio is shown to the flow rate. For Newtonian glycerin flow with 32 throttling ratio the streak length is long at slow flow rate. With increasing flow rate, the length becomes shorter and approaches a constant length. For 0.05\% PAA solution having weak viscoelastic property, the streak length takes a constant value about equal to the constant length for the glycerin flow. At high polymer concentration, the streak line ratio increases with increasing flow rate and reaches a constant length. High polymer concentration and large throttling ratio make the flow
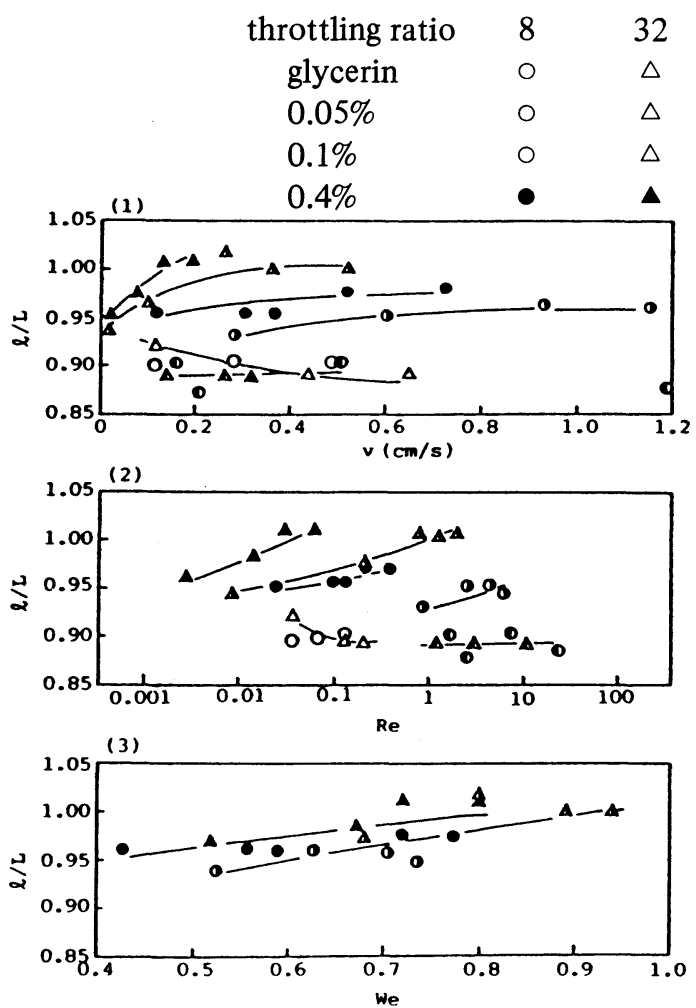

Fig. 12 Length of streak line 
rate reach a constant length at slow flow.

In Fig. 12(2) the length ratio is shown to Reynolds number, and in Fig. 12(3) to Weissenberg number (We) defined as follows:

$$
\begin{aligned}
& W_{e}=\lambda \frac{v_{0}}{D_{0}} \ldots \ldots \ldots \ldots \ldots \ldots \ldots \ldots \ldots \ldots \ldots \ldots \ldots \ldots \ldots \ldots
\end{aligned}
$$

where, $D_{0}$ is the outlet tube diameter and $v_{0}$ the mean velocity in the tube. There are two reasons why the variables of the outlet are adopted. One is because two kinds of outlet tubes are used, and the other is because the elastic effect is influenced by the applied strain. $\lambda$ is the relaxation time shown in equation (4) by Maxwell model. Shear rate $\dot{\gamma}$ is calculated at the radius of the tube where the flow rate equals to mean velocity. The value of the viscosity $\eta$ and the first normal differences $N_{1}$ are obtained from Bewersdorff's measurements ${ }^{[19]}$.

Two curves seen in Fig. 12(1) obtained from low concentration $0.05 \%$ PAA solution and glycerin flow become one continuous curve by rearranging with Reynolds number. This shows that for $0.05 \%$ PAA solution the flow behavior resembles to Newtonian flow and the elastic effect can be ignored. In the case of high concentration polymer solution, these length ratio curves are separate.

Shown in Fig. 12(3) is the result obtained by rearranging with Weissenburg number for $0.1 \%$ and $0.4 \%$ PAA solution. The plots of length ratio are classified in two curves with different polymer concentration but of the same throttling ratio. Two curves lie near compared with that for rearranging with Reynolds number or with mean velocity.

Similar results are obtained from the observation of the streaks started from $A$ or $B$ etc. Hence, the reduction of the swell of the streamline with increasing flow rate seems by the inertial effect, and at high flow rate the growth and the expansion of the swell with increasing flow rate seems by the elastic effect.

\section{Shape of Circulating Secondary Flow}

Growth of the circulating secondary flow for viscoelastic fluids can be observed two dimensionally as shown in Fig. 6. The secondary flow is expected to locate widely on the circumference of the exit. Shown in Fig. 13 are the flows of colored tracer in the secondary flow. Fig. $13(1)$ is taken shortly after the tracer entering the secondary flow. Fig. 13(2) is after a few minute. These two pictures are taken along $z$ direction and other (3) and (4) are along $y$ direction. Pictures along $y$ direction show that the circulating secondary flow expands on the circumference of the exit.

Shown in Fig. 14 is the schematic diagram of the

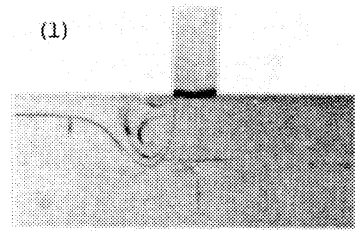

(3)

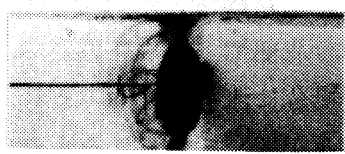

(4)

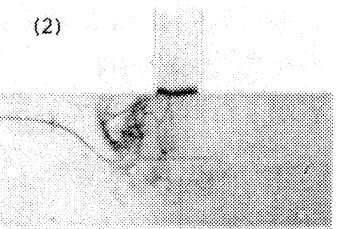

4)
Fig. 13 Tracer fluid in circulating secondary flow for PAA $0.4 \%$ solution. $v=0.21 \mathrm{~cm} / \mathrm{s}$ and throttling ratio $=32$. Upper pictures are along $z$ direction and lower along $y$ direction

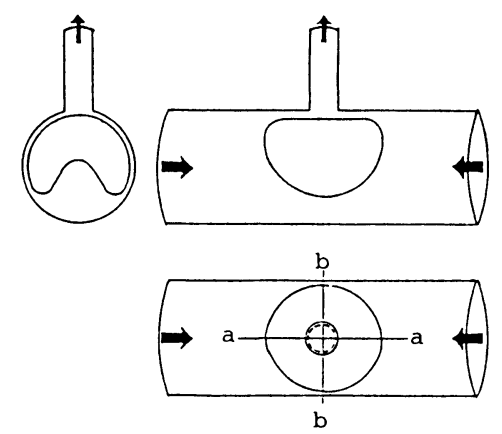

(1)

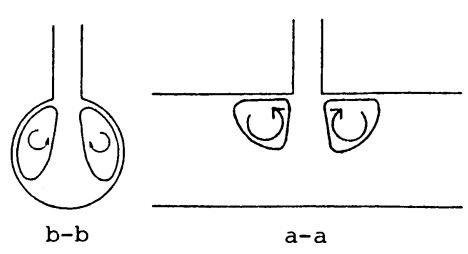

(2)

Fig. 14 Schematic diagram of circulating secondary flow for a T-shaped flow. Upper is external, and lower sectional appearance.

circulating secondary flow at the combining part of the T-shaped tube. Circulating secondary flow seen in $x$ direction expands along the wall of the inlet tube from the entrance of the outlet tube. That seen in $z$ direction is a triangle having dull corners. That in $y$ direction shows a torus on the exit. Domain of the secondary flow in a-a section is smaller than that in $b-b$ because of the main flow.

\section{Conclusion}

With a T-shaped conduit, viscoelastic combining flow is observed two and three dimensionally and compared with Newtonian flow. 
For slow flow with viscoelastic and Newtonian fluids, swelling of the streamline is observed at the combining part in the section along the inlet tube. The swelling becomes smaller with increasing flow rate, and finally disappeared to have a parallel flow. For faster viscoelastic flow, a circulating secondary flow grows at the upstream side of the reentrant corner of the combining part. Same observation was carried out for a $\mathrm{T}$-shaped rectangular conduit.

The size of the circulating secondary flow increases with increasing flow rate. The growth of the domain is clear at high polymer concentration or at high throttling ratio of the tube. This is the result by the elastic property of the polymer solution.

The flow at the combining part is discussed three dimensionally by observing the tracers through six points set up in the cross section of the inlet tube. The streak line length in the fixed section at the combining part is long when there is the swell. For Newtonian fluid and low concentration polymer solution, the streak line is long at slow flow rate and becomes shorter with increasing flow rate and approaches a constant length. Two curves obtained with Newtonian and polymer solutions become to one by arranging with Reynolds number. For polymer solution, the streak line become longer with increasing flow rate, because a circulating secondary flow grows at the combining corner. In this case, the length ratio curves obtained by rearranging with Reynolds number or mean velocity depend on the polymer concentration and the throttling ratio of the tube. But their curves almost gather to a continuous curve by arranging with Weissenberg number. Thus, the decrease of the swelling domain at slow flow rate is the result by the inertial effect, and the growth and expansion of the swell at high flow rate is by the elastic effect.

The circulating secondary flow appearing at the combining part locates on the circumference of the entrance to the exit tube, and its size is rather small in the main flow direction, but large along the wall direction.
A part of this report was presented at the 38th annual meeting of Textile Machinery Society of Japan, Osaka, June, 1985.

\section{References}

[1] T. Nishimura, K. Nakamura, A. Horikawa; Sen-i Kikai Gakkai Ronbunsyu, 36, T66 (1983)

[2] T. J. Pedley, R. C. Schroter and M. F. Sudlow; J. Fluid Mech., 46, Part 2, 365 (1971)

[3] C. M. Rodkiewicz and C. L. Roussel; J. Fluids Eng., 95, 108 (1973)

[4] S. Oka; Zairyo, 29, 321 (1980)

[5] F. R. Haselton and P. W. Scherer; J. Fluid Mech., 123, 315 (1982)

[6] H. Ito, M. Sato, K. Oka; Nihon Kikai Gakkai Ronbunsyu (Part 2), 44, 3902 (1978)

[7] H. Ito, M. Sato, K. Oka; Nihon Kikai Gakkai Ronbunsyu (Part B), 47, 214 (1981)

[8] E. B. Bagley and A. M. Birks; J. Appl. Phys., 31, 556 (1960)

[9] T. F. Ballenger and J. L. White; Chem. Eng. Sci., 25, 1191 (1970)

[10] Y. Oyanagi, Y. Yamaguti; Zairyo, 20, 659 (1971)

[11] T. F. Ballenger, I-J. Chen, J. W. Crowder, G. E. Hagler and D. C. Bouge; Trans. Sci. Rheo., 15:2, 195 (1971)

[12] T. F. Ballenger and J. L. White; J. Appl. Polym. Soc., 15, 1949 (1971)

[13] H. P. Hurlimann und W. Knappe; Rheol. Acta., 11, 292 (1972)

[14] T. Nishimura, K. Saijo, T. Soen; Sen-i Gakkaisi, 33, T246 (1977)

[15] M. J. Rudd; Nature, 224, 587 (1969)

[16] W. A. Hanson, D. F. Jankowski and N. S. Berman; Phys. Fluid, 12, 2702 (1969)

[17] J. S. Chung and W. P. Graebel; Phys. Fluid 15, 546 (1972)

[18] M. J. Rudd; J. Fluid., 51, 673 (1972)

[19] H. W. Bewersdorff; Rehol. Acta., 23, 522 (1984) 